\title{
Sustainable Resource Planning in Energy Markets
}

\author{
Saeed Kamalinia ${ }^{(1)}$, Mohammad Shahidehpour ${ }^{(2)}$, Lei Wu ${ }^{(3)}$ \\ ${ }^{(1)}$ Power Systems Solutions, S\&C Electric Company \\ ${ }^{(2)}$ ECE Department, Illinois Institute of Technology \\ ${ }^{(3)}$ ECE Department, Clarkson University
}

\begin{abstract}
This study investigates the role of sustainable energy volatility in a market participant's competitive expansion planning problem. The incomplete information non-cooperative game-theoretic method is utilized in which each generation company (GENCO) perceives strategies of other market participants in order to make a decision on its strategic generation capacity expansion. Sustainable generation incentives, carbon emission penalties, and fuel price forecast errors are considered in the strategic decisions. The market clearing process for energy and reserves is simulated by each GENCO for deriving generation expansion decisions. A merit criterion (i.e., the utility value) is proposed for a more realistic calculation of the expected payoff of a GENCO with sustainable energy resources. Finally, the impact of transmission constraints is investigated on the GENCO's expansion planning decision. The case studies illustrate the effectiveness of the proposed method.
\end{abstract}

Keywords: Sustainable energy resource; expansion planning; energy market; generation company; game theory.

NOMENCLATURE

The symbols used in this paper are classified into indices, parameters, decision variables, and matrices and vectors as follows.

\section{Indices}

C

E

$g$

$i$

j

$m, n$

NW

$s$

$t$

W

\section{Parameters}

$\alpha$

$\sigma$

CGW

CGNW

$\mathrm{Cap}_{\max }$

$D T_{t}$
Superscript index for candidate generation units or transmission lines

Superscript index for existing units or lines

Subscript for generation companies (GENCOs)

Index for generating units

Index for transmission lines

Subscript for bus numbers

Index for thermal units

Index for scenarios

Subscript for time periods

Index for wind units

(C) 2014. This manuscript version is made available under the Elsevier user license http://www.elsevier.com/open-access/userlicense/1.0/ 


\begin{tabular}{|c|c|}
\hline$E G_{g}$ & Number of committed generating units of GENCO $g$ \\
\hline$E(\cdot)$ & Expected value \\
\hline$E C(\cdot)$ & Emission cost \\
\hline FOR & Forced outage rate \\
\hline$I C(\cdot)$ & Levelized investment cost \\
\hline $\operatorname{INC}(\cdot)$ & Incentive payment \\
\hline$N B$ & Number of buses \\
\hline$N G C$ & Number of GENCOs \\
\hline$N T$ & Number of planning periods \\
\hline$O(\cdot)$ & Outage cost \\
\hline$O C$ & Average operating cost \\
\hline$p\left(t_{i}\right)$ & Probability when participant $i$ 's type is $t_{i}$ \\
\hline$p\left(\mathbf{t}_{-i} \mid t_{i}\right)$ & Conditional probability when GENCO $i$ 's type is $t_{i}$ and its opponents' type is $\mathbf{t}_{-i}$ \\
\hline$p(s)$ & Probability of scenario s \\
\hline$r$ & Investment payoff \\
\hline$R C$ & Reserve cost \\
\hline$R P$ & Revenue from the energy market \\
\hline$P R$ & Revenue from the reserve market \\
\hline$S c$ & Planning scenario \\
\hline$S_{o}$ & Opponents' strategy \\
\hline$S_{s}$ & Self-strategy \\
\hline$S_{t}$ & GENCOs’ final expansion strategy \\
\hline$t y_{i}$ & GENCO $i$ 's type \\
\hline $\mathbf{t y}-i$ & Type of GENCO $i$ 's opponents \\
\hline $\mathbf{T Y} \mathbf{Y}_{i}$ & The set of GENCO $i$ 's types \\
\hline $\mathbf{T} \mathbf{Y}_{-i}$ & The set of types of GENCO $i$ 's opponents \\
\hline$U$ & Utility value \\
\hline$U Y_{m n, t s}$ & Status of transmission line $m-n$ in time $t$ of scenario $s ; 0$ if on outage, otherwise 1 \\
\hline$U X_{g t s}$ & Status of GENCO $g$ in time $t$ of scenario $s ; 0$ if on outage, otherwise 1 \\
\hline$Y_{j t}$ & Installation status of transmission line $j$ in year $t$ \\
\hline$\gamma_{j}$ & Phase shifter angle on line $j$ \\
\hline
\end{tabular}

\section{Decision Variables}

$\begin{array}{ll}P C & \text { Load shedding quantity } \\ P G & \text { Dispatched capacity in the energy market } \\ P G_{\max }^{C} & \text { Capacity of a candidate generation unit } \\ P L & \text { Power flow of a transmission line } \\ P R & \text { Reserve capacity bid to the ancillary service market } \\ Q_{R} & \text { Reserve capacity price } \\ X & \text { Generation unit's expansion type } \\ \theta & \text { Bus angle } \\ \lambda_{p}, \lambda_{R} & \text { Lagrangian multipliers }\end{array}$

\section{Matrices and Vectors}

$\begin{array}{ll}\text { A } & \text { Bus-unit incidence matrix } \\ \text { B } & \text { Bus-load incidence matrix } \\ \mathbf{K} & \text { Bus-branch incidence matrix }\end{array}$




$\begin{array}{ll}\text { P } & \text { Real power output vector } \\ \text { PC } & \text { Load shedding vector } \\ \text { PD } & \text { Load vector } \\ \text { PL } & \text { Real power flow vector } \\ \text { UY } & \text { Transmission line availability status vector }\end{array}$

\section{INTRODUCTION}

Market participants seek to maximize their payoffs by optimizing their investment on sustainable energy resources [1]. A market participant's decision is based on its own investment strategies as well as its imperfect knowledge of other participants' strategies. Therefore, self-interested stakeholders learn and adapt to the perceived behaviors of other participants in order to maximize their payoffs in competitive markets. In a market, the independent system operator's (ISO's) objective is to supply the demand at the minimum cost while maintaining a pre-specified level of system reliability.

Generation expansion planning (GEP) has been broadly examined in the literature. Using the Benders decomposition method, a profit-based generation resource planning was discussed in [2], which considered the economics and the reliability of market operation. Lagrangian relaxation algorithm was applied in [3] to relax complicated coupling constraints into the objective function of planning problems and obtain locational capacity signals. An agent-based model was applied in [4] to simulate an expansion planning approach which took into account interactions among multiple agents in electricity markets. The paper considered an incomplete information model where individual generators (agents) do not have information of its opponents. One of the early studies on the application of non-cooperative game theory was presented in [5]. The paper simplified the problem by assuming the availability of complete information to all opponents. The competitive behavior of GENCOs in an electricity market was considered in [6] by applying a game theoretic method, and a co-evolutionary algorithm was proposed to model GENCOs as agents. Applying artificial intelligence algorithms may lead to non-convergence or sub-optimal results in case of large study systems with numerous market participants, while the simulation time is always a concern when using such methods. Furthermore, GENCOs can receive misleading profit signals by neglecting expected transmission expansions in the system and/or uncertain factors. A deterministic model was applied in [7], which used game theory to investigate the strategic interaction among GENCOs and transmission companies (TRANSCOs) in a restructured market environment. A multi-agent expansion model in [8] investigated interactions among self-interested GENCOs in electricity markets. A hybrid GEP algorithm that utilized a combination of game theory and genetic algorithm was presented in [9]. In practice, expansion planning forms a first-price sealed-bid auction [10] problem in which individual market participants have a perfect knowledge of their own payoffs and strategies, but lack such a complete information on other participants [11]. The expansion planning problem was modeled as an incomplete information environment [12], in which a participant could potentially lower its payoff if it does not consider the opponents' strategies. A stochastic mixed-integer linear programming (MILP) approach was applied in [13] to form a structure for making profit-maximization based expansion decision in a liberalized market. Reference [13] also performed a 
thorough literature review in the area of generation investment.

Sustainable resources are abundant, environment friendly, and volatile [14]. Sustainability prohibits the depletion of energy resources and the accumulation of residues [15], and is subjected to incentive credits in many energy markets. Higher fuel prices, long permitting process, and environmental constraints have limited the planning options for large fossil-fuel power plants. A multi-objective model for the hybrid generation and transmission corridors expansion planning incorporating certain sustainable energy resources was presented in [16]. The model is applicable to centralized power systems for minimizing the total investment and operating costs. Hybrid expansion planning of generation and transmission was examined in [17]. Results of the study in [17] clearly indicate that despite decisions of generation and transmission planning are made by independent entities, the physical connection between generation and transmission expansion necessitates considering the mutual impacts of these sectors. The approach in [18] showed that a mix of sustainable energy resources could lead to lower generation costs despite their higher investment costs. To overcome such constraints, participants are increasingly looking into sustainable options for optimizing generation strategies and diversifying generation portfolios. In the United States, renewable portfolio standards (RPSs) are mandated in many states. In addition, production tax credit (PTC) and investment tax credit (ITC) are made available to encourage the development of various types of sustainable generation resources. Complementary technologic and economic tools are also being utilized to mitigate the carbon emission of electrical systems [19]. Carbon emission tax policies have been initiated by several ISOs [20, 21]. Sustainable generation units, e.g., wind generators, also provide an opportunity for GENCOs to arbitrage the emission allowance. However, such units introduce operation challenges due to their volatile and uncertain nature. Therefore, the reliability of power systems with a substantial integration of wind generation must be investigated [22].

In a competitive capacity expansion strategy, GENCOs would optimize the share of sustainable generation in their generation portfolios. When considering sustainable energy resources, distinct regions are prioritized as, for example, wind generation is dependent significantly on wind speed and direction. Long-term economic impacts of wind energy was investigated in [23] in a power system consisted of conventional generating units and wind farms. The results of studies in [23] verify the necessity of applying stochastic approach for wind energy analyses. Furthermore, the paper unveiled a key conclusion that as the wind penetration increases, wind farm revenue may decreases. This is a vital concern in generation expansion planning in the investor's viewpoint that considers wind energy as an expansion option. While the inclusion of sustainable energy resources can significantly alter the long-term GEP, it is imperative to consider short-term operational issues in such studies [24].

In this paper, we consider the GEP with incomplete information in which a market participant would reduce the financial risk of investment by using the attractive features of sustainable energy resources and considering expected transmission network expansions in the area. In the proposed model, we considered the volatility and the uncertainty of wind generators, which could affect a GENCO's expected payoff. As shown in [6], GENCOs can better project their payoffs if they consider opponents' 
behaviors by using the game-theoretic approach. The salient distinction of this paper as compared to previous work in [6] is investigating how sustainable energy resources would change the competition process, while considering the expected transmission network expansion. The major contribution of this paper is applying a hybrid generation-transmission expansion planning model, which significantly helps a GENCO to avoid highly unexpected outcomes. Compared to conventional optimization methods that only maximize expected profit or minimize social cost, this paper includes the utility value in the objective, which takes into account merits of having various generation portfolios and minimizes their associated risk of investment. In the game-theoretic approach, as each participant intends to maximize its surplus, the inclusion of sustainable generation would profoundly change the results. The salient feature of the proposed method is that GENCOs are modeled as independent agents, which could compete in a volatile market for maximizing their utility values by using sustainable energy options. The motivation behind this paper is to investigate an approach to tackle emerging challenges of considering sustainable generation in a participant expansion plan as follows.

- The increasing uncertainty in participant payoffs because of the unpredictability of sustainable generation;

- The necessity to consider incentives for sustainable energy generation and emission costs in calculating participants' payoffs;

- The intermittency of sustainable energy resources;

- Application of the stochastic approach for evaluating the unpredictability of opponent payoffs and commodity values;

- The inclusion of sustainable energy resources will add more complexity to game-theoretic model because of the variety of available options to participants for generation expansion;

- The long-term study of sustainable energy resources is required because of high initial investment costs and low operating costs, which would accordingly result in extended payback periods;

- The proposed stochastic model investigates the fuel cost uncertainty of fossil-based generations as compared to the modeling of fuel-free sustainable generation.

Since the proposed model represents a long-term planning problem, stochastic programming is utilized to consider uncertainties. The random variables introduced in our model are wind and load forecast errors as well as the availability of system components (i.e., transmission lines and generating units). We consider a set of possible scenarios based on the Monte Carlo simulation method. Fuel cost uncertainty is also modeled by implementing the utility value approach.

In the proposed model, economically independent GENCOs make generation expansion decisions while considering a mix of generation expansion options (i.e., sustainable and conventional generations). We consider the proposed options for transmission expansion in the GENCOs strategic plan. Numerical experiments discuss the impact of transmission constraints on the competitive GEP. 
The rest of this paper is organized as follows. Section 2 describes the proposed expansion planning process. Section 3 elaborates the description of the proposed GENCO's strategic expansion model and discusses the mathematical formulation and the solution methodology. The case study is analyzed in Section 4. Lastly, in Section 5, the conclusions drawn from the studies are provided.

\section{The PROPOSED GENCO’s GEP FoRMULATION AND SOLUTION METHODOLOGY}

In the following sections we review GENCO's planning model and the proposed approach for solving the problem.

\subsection{GENCO's Generation Expansion Planning Model}

In our model, each GENCO would consider a range of expansion strategies, including sustainable generation options, and maximize its utility value by modeling possible expansion decisions of other GENCOs. The concept of the utility value will be described in more details in the following section. The role of transmission constraints in the GENCOs' strategic planning is also modeled. Fig. 1 shows the GEP's simulation framework with incomplete information. In this model, each GENCO prepares a planning proposal based on its own utility value maximization.

The proposed planning model in Fig. 1 forms a bi-level optimization problem. In the upper level, each participant identifies its optimal planning among feasible expansion options. The lower level is associated with the simulation of perceived market clearing problem. In Fig. 1, the solution yields the equilibrium result if participants reach their individual maximum expected payoffs and do not alter the proposed GEP [25].

\subsection{Proposed Formulation And Solution Methodology}

The following assumptions are considered in the proposed model:

- $\quad$ Each GENCO acts as a rational player by maximizing its annual levelized expected payoff

- $\quad$ Each GENCO has a finite set of GEP strategies

- Ramping rates and minimum ON/OFF time constraints of individual generating units are excluded in the long-term GEP study

- The output of the proposed GEP approach would satisfy operating constraints (generation units, nodal power balance, and reserve margin) and system constraints (possible candidate sites and the limits on generation expansion)

- Wind energy generation, as the most dominant sustainable energy resource, is solely considered in the formulation without losing the generality of the proposed approach

- The operation cost of wind generation is negligible

- The present value of the capital cost for a GENCO's capacity expansion over its economic life is converted to annual costs 
- Wind generators operate at their maximum dispatchable capacity by only participating in energy markets, while thermal units also participate in reserve markets

- Incentive payment to participating wind units is based on their dispatched capacity

- Hourly load and wind data are used in the market simulations

The details of the proposed approach are discussed as follows.

\subsubsection{GENCO's GEP Model}

Each GENCO would initiate its GEP by considering historical data, market trends, published information on contracts, and planning parameters. GENCOs have options to make. The GENCO's capacity expansion objective is to maximize its expected payoff with minimum financial risk. In this paper, the financial risk for thermal GEP would address volatility and forecast errors in fuel prices. Nevertheless, wind generation, which would not incur any fuel price risks, may also result in lower than expected payoff because of its unpredictable nature. GEP is modeled by considering the utility value concept, which is detailed in the following. Wind generation uncertainty is considered via scenarios using the Monte Carlo method [26-30].

Each GENCO assigns weights to its strategies according to a joint probability distribution. A GENCO would estimate other GENCOs' types and joint probability distributions as part of its strategy. Each GENCO selects at a time only one option for GEP among wind $\left(X_{W}=1, X_{N W}=0\right)$, thermal $\left(X_{W}=0, X_{N W}=1\right)$, or "no-investment" $\left(X_{W}=0, X_{N W}=0\right)$ strategies. Here, a simultaneous (hybrid) wind-thermal planning option $\left(\mathrm{X}_{\mathrm{W}}=1, \mathrm{X}_{\mathrm{NW}}=1\right)$ is not considered. A GENCO may defer any investments at a given time (i.e., "no-investment" strategy), if it cannot achieve its minimum expected profit through any available expansion strategies at that time. Accordingly, the GENCO may revise the proposed expansion options before applying the approach again.

A GENCO is seeking higher expected payoffs and lower financial risks. Therefore, the objective (utility) function would have the form $\mathrm{U}$ (payoff, risk). The utility function (value) presented in [31] assumes to present by an order-2 approximation as (1).

$\mathrm{U}\left(\mathrm{E}(\mathrm{r}), \sigma^{2}\right)=\mathrm{E}(\mathrm{r})-\xi \mathrm{A} \sigma^{2}$

in which we consider the following possible situations:

- If $\mathrm{A}>0$, risk-averse participant;

- If $\mathrm{A}=0$, risk-neutral participant $\rightarrow \mathrm{U}=\mathrm{E}(\mathrm{r})$;

- If $\mathrm{A}<0$, risk-lover participant.

' $A$ ' is the participant's degree of risk aversion. Higher 'A' implies more risk averse, and vice versa (usually set to integer values less than 6). Fig. 2 shows the variation of utility function for a sample project with different degree of risk aversion of participants. $\xi$ is a normalizing factor to reduce the size of the variance, $\sigma^{2}$, which is a measure on the volatility of the investment 
and therefore its risk. By setting $\xi=0.005$, this equation is also normalized, so that the result is a yield percentage that can be compared to investment payoffs, which allows the utility score to be directly compared to other investment payoffs.

The GENCO assigns welfare or utility value to evaluate its investment portfolio based on expected payoff and associated financial risks. The above rationale application of utility value is adopted in the following GEP model. One reasonable utility for a portfolio with expected payoff $E(r)$ and variance of payoff $\sigma^{2}$ is described in (2) [31].

$U=E(r)-0.005 A \sigma^{2}$

where $\mathrm{U}$ is the utility value, $A$ is an index for the participant's risk aversion which depends on the participant's risk sensitivity, 0.005 is a scaling factor that expresses the expected payoff and the standard deviation as percentages rather than decimals, and $\sigma$ is the standard deviation of uncertain factors (e.g., fuel price).

The utility value is a means of ranking portfolios. Higher utility values are assigned to portfolios with more attractive riskpayoff profiles. That is, portfolios receive higher utility values for higher expected payoffs.

The GENCO's optimization problem is formulated as (3).

$$
\operatorname{Max} U_{G i}=E\left(r_{i g, t_{i}}\right)-0.005 A_{g} \sigma_{g}^{2}
$$

where the expected payoff for GENCO $g$, with the unit type $t y_{\mathrm{i}}$ and the opponents' type of $t y_{-\mathrm{i}}$ is given as (4)-(5).

$$
\begin{aligned}
& E\left(r_{i g, t y_{i}}\right)=\sum_{s} \sum_{t y_{i} \in T Y_{i}} \sum_{\mathbf{t y}_{-i} \in T Y_{-i}} r_{i g, t y_{i}}\left(s, t y_{i}, t y_{-i}\right) \cdot p\left(\mathbf{t y} \mathbf{- i}_{-i} \mid t y_{i}\right) \cdot p\left(t y_{i}\right) \cdot p(s) \\
& r_{g i, t y_{i}}\left(s, t y_{i}, t y_{-i}\right)=R P\left(s, t y_{i}, \mathbf{t y}_{-i}\right)+R R\left(s, t y_{i}, \mathbf{t y} \mathbf{y}_{-i}\right)+I N C\left(s, t y_{i}, \mathbf{t y} \mathbf{y}_{-i}\right)-I C\left(t y_{i}\right)-O\left(s, t y_{i}, \mathbf{t y} \mathbf{y}_{-i}\right)-E C\left(s, t y_{i}, \mathbf{t y} \mathbf{y}_{-i}\right)
\end{aligned}
$$

The GENCO's hourly load and unit outage scenarios are considered in (4). In (5) $R P(\cdot)$ and $R R(\cdot)$ are GENCO's revenue from energy and reserve markets, respectively, and $I N C(\cdot)$ represents tax discounts and sustainable energy incentives for GEP. We consider the emission cost, $E C(\cdot)$, in the objective function rather than a constraint so that GENCOs can arbitrage emission allowances [32]. GENCO's mainstream revenue comes from energy market as detailed in (6):

$$
\begin{aligned}
R P\left(s, t y_{i}, t y_{-i}\right)= & \sum_{t=1}^{N T} \sum_{i=1}^{E G_{g}} D T_{t}\left(L M P_{i g t}^{E, N W}\left(s, t y_{i}, \mathbf{t y}_{-i}\right)-O C_{i g t}^{E, N W}\left(t y_{i}\right)\right) P G_{i g t}^{E, N W}\left(s, t y_{i}, \mathbf{t y}-i\right)+ \\
& \sum_{t=1}^{N T} \sum_{i=1}^{C G N W_{g}} D T_{t}\left(L M P_{i g t}^{C, N W}\left(s, t y_{i}, \mathbf{t y}_{-i}\right)-O C_{i g t}^{C, N W}\left(t y_{i}\right)\right) P G_{i g t}^{C, N W}\left(s, t y_{i}, \mathbf{t y}_{-i}\right)+ \\
& \sum_{t=1}^{N T} \sum_{i=1}^{C G W_{g}} D T_{t}\left(L M P_{i g t}^{C, W}\left(s, t y_{i}, \mathbf{t y}_{-i}\right)-O C_{i g t}^{C, W}\left(t y_{i}\right)\right) P G_{i g t}^{C, W}\left(s, t y_{i}, \mathbf{t y}_{-i}\right)
\end{aligned}
$$

Where,

$$
P G_{g}\left(b, s, t y_{i}, \mathbf{t y}_{-i}\right)=\left[\begin{array}{l}
P G_{g t}^{N W}\left(b, s, t y_{i}, \mathbf{t y}_{-i}\right) \\
P G_{g t}^{W}\left(b, s, t y_{i}, \mathbf{t y}_{-i}\right) \\
P G_{g t}^{E, N W}\left(b, s, t y_{i}, \mathbf{t y}_{-i}\right)
\end{array}\right]^{t} \quad \text { and } O C_{g}\left(t y_{i}\right)=\left[\begin{array}{l}
O C_{g}^{C, N W}\left(t y_{i}\right) \\
O C_{g}^{C, W}\left(t y_{i}\right) \\
O C_{g}^{E, N W}
\end{array}\right]
$$


Additional revenues from incentive credits (i.e. for wind generation) and reserve market (i.e. currently only for non-wind generation) are available for GENCO, as shown in (7)-(8).

$$
\begin{aligned}
& R R\left(s, t y_{i}, t y_{-i}\right)=\sum_{t=1}^{N T} \sum_{i=1}^{E G_{g}} D T_{t} Q R_{i g t}^{E, N W} P R_{i g t}^{E, N W}\left(s, t y_{i}, \mathbf{t y}_{-i}\right)+\sum_{t=1}^{N T} \sum_{i=1}^{C G N W_{g}} D T_{t} Q R_{i g t}^{C, N W} P R_{i g t}^{C, N W}\left(s, t y_{i}, \mathbf{t y}_{-i}\right) \\
& I N C\left(s, t y_{i}, \mathbf{t y}_{-i}\right)=\sum_{t=1}^{N T} \sum_{i=1}^{C G W_{g}} D T_{t}\left(\alpha_{i g t} P G_{i g t s}^{C, W}\left(t y_{i}\right)\right)
\end{aligned}
$$

Various costs of generation expansion planning are listed in (9)-(11):

$I C\left(t_{i}\right)=\sum_{t=1}^{N T} \sum_{i=1}^{C G N W_{g}}\left(I C_{i g t}^{N W}\left(t y_{i}\right) P G_{\max , i g}^{C, N W}\right)+\sum_{t=1}^{N T} \sum_{i=1}^{C G W_{g}}\left(I C_{i g t}^{W}\left(t y_{i}\right) P G_{\max , i g}^{C, W}\right)$

$O\left(s, t y_{i}, \mathbf{t y}_{-i}\right)=\sum_{s} \sum_{i} \sum_{t} F_{i} O R_{i}\left(R P\left(s, t y_{i}, t y_{-i}\right)+R R\left(s, t y_{i}, t y_{-i}\right)\right)$

$E C\left(s, t y_{i}, \mathbf{t y}_{-i}\right)=\sum_{t=1}^{N T} \sum_{i=1}^{E G_{g}} D T_{t}\left(E C_{i g t} P G_{i g t}^{E, N W}\left(s, t y_{i}, \mathbf{t y}_{-i}\right)\right)+\sum_{t=1}^{N T} \sum_{i=1}^{C G_{g}} D T_{t}\left(E C_{i g t} \cdot P G_{i g t}^{C, N W}\left(s, t y_{i}, \mathbf{t y}_{-i}\right)\right)$

The constraints applied in this stage are the capacity limit for each expansion strategies (12)-(13):

$0 \leq P G_{\max i g}^{C, N W}\left(s, t y_{i}, \mathbf{t y}_{-i}\right) \leq \operatorname{Cap}_{\max i g}^{C, N W}\left(t y_{i}\right) \cdot X_{N W}$

$0 \leq P G_{\max , i g}^{C, W}\left(s, t y_{i}, \mathbf{t y}_{-i}\right) \leq \operatorname{Cap}_{\max , \mathrm{ig}}^{C, W}\left(t y_{i}\right) \cdot X_{W}$

The GENCO's decision variables are the capacities (i.e., $P G_{\max , g}^{C, N W}\left(t y_{i}\right)$ and $\left.P G_{\max , g}^{C, W}\left(t y_{i}\right)\right)$ and the unit types (i.e., $\mathrm{X}_{\mathrm{W}}$ and $\mathrm{X}_{\mathrm{NW}}$ ), which will be considered in market clearing process in the below Section 2.2.2.

\subsubsection{Market Clearing Simulation}

The market clearing objective simulated by GENCO $g$ (14) would minimize the costs of load shedding, operation, and reserves, subject to nodal load balance constraint (15), line flow limits (16), load curtailment limits (17), system reserve requirement (18), generation limits (19)-(21), DC power flow (22)-(23), and the reference bus angle constraint (24) [33].

$\operatorname{Min} \sum_{g=1}^{N G C}\left[\left(P G_{g}\left(s, t_{i}, \mathbf{t}_{-i}\right) O C_{g}\left(t_{i}\right)\right)+\left(P R_{g}\left(s, t_{i}, \mathbf{t}_{-i}\right) R C_{g}\left(t_{i}\right)\right)\right]+\sum_{l=1}^{N D}\left(P C_{l}\left(s, t_{i}, \mathbf{t}_{-i}\right) L S_{l}\right)$

s.t.

$\mathbf{K} \cdot \mathbf{P L}-\mathbf{A} \cdot \mathbf{P}+\mathbf{B} \cdot(\mathbf{P D}-\mathbf{P C})=0 \quad \lambda_{P}$

$-\mathbf{P L}_{\max } \cdot \mathbf{U Y} \leq \mathbf{P L} \leq \mathbf{P} \mathbf{L}_{\max } \cdot \mathbf{U Y}$

$0 \leq P C_{t}\left(s, t y_{i}, \mathbf{t y}_{-i}\right) \leq P D_{t}$ 


$$
\begin{aligned}
& \sum_{g=1}^{N G} P R_{g}^{N W}\left(s, t_{i}, \mathbf{t y}_{-i}\right) \geq 0.1\left(P D_{s t}-P C_{l}\left(s, t y_{i}, \mathbf{t y}_{-i}\right)\right) \quad \lambda_{R} \\
& P G_{g t}^{C, N W}\left(s, t_{i}, \mathbf{t y}_{-i}\right)+P R_{g t}^{C, N W}\left(s, t y_{i}, \mathbf{t y}_{-i}\right) \leq P G_{\max , g}^{C, N W}\left(t y_{i}\right) \cdot X_{N W} \cdot U X_{g t s} \\
& 0 \leq P G_{g t}^{W}\left(s, t_{i}, \mathbf{t}_{-i}\right) \leq P G_{\max , g t s}^{W}\left(t_{i}\right) X_{w} U X_{g t s} \\
& 0 \leq P G_{g t}^{E, N W}\left(s, t_{i}, \mathbf{t}_{-i}\right)+P R_{g t}^{E, N W}\left(s, t_{i}, \mathbf{t}_{-i}\right) \leq P G_{\max , g}^{E} U X_{g t s} \\
& P L_{j t s}-\frac{\left(\theta_{m, t s}-\theta_{n, t s}-\gamma_{j}\right)}{x_{j}}+M\left(1-U Y_{j t s}\right) \geq 0 \\
& P L_{j t s}-\frac{\left(\theta_{m, t s}-\theta_{n, t s}-\gamma_{j}\right)}{x_{j}}-M\left(1-U Y_{j t s}\right) \leq 0 \\
& \theta_{\text {ref }}=0
\end{aligned}
$$

The uncertainty is characterized in multiple scenarios in this paper. $P G_{\max \text { gts }}^{W}$ is the maximum available wind power at hour $t$ and scenario $s$. In this study, the hourly wind power forecast is assumed to be given in each scenario [34]. The transmission network augmentation is represented by (25)-(27).

$$
\begin{aligned}
& P L_{j t}^{C}-\frac{\left(\theta_{m b t}^{C}-\theta_{n b t}^{C}-\gamma_{j}\right)}{x_{j}}-M\left(1-Y_{j t}\right)-M\left(1-U Y_{j t s}^{C}\right) \leq 0 \\
& P L_{j t}^{C}-\frac{\left(\theta_{m b t}^{C}-\theta_{n b t}^{C}-\gamma_{j}\right)}{x_{j}}+M\left(1-Y_{j t}\right)+M\left(1-U Y_{j t s}^{C}\right) \geq 0 \\
& -P L_{\max , j}^{C} Y_{j t} U Y_{j b t}^{C} \leq P L_{j b t}^{C} \leq P L_{\max , j}^{C} Y_{j t} U Y_{j b t}^{C}
\end{aligned}
$$

GENCOs may obtain the transmission information from the ISO or make their own estimates based on market trends. It is assumed that the market clearing problem has a feasible solution (i.e., available generation capacity is larger than the total demand). However, a GENCO may either increase perceived generation expansion capacity or consider load shedding, if there is not enough generation capacities to supply the demand. Furthermore, a GEP strategy would be eliminated if the transmission capacity provided in that strategy is insufficient. In this case, the GENCO may revise the GEP proposal by choosing another option for interconnection.

\subsubsection{Generalized Formulation}

Each GENCO forms its desired feasible range of expansion strategies by anticipating the opponents' strategies according to its best knowledge. In the next step, GENCOs evaluate their plans and select the best strategy according to the simulated markets. A plan will be selected only if it shows the maximum utility value in all scenarios $(S c)$ during the planning period $(t)$. GENCOs 
consider expected payoffs along with their associated level of risks. Therefore, GENCOs make final planning decisions based on the utility value in each strategy and the signals obtained from the market clearing simulation. The GEP problem is a two-level optimization. The upper level problem is formulated as (28).

$\max U($ profit, risk)

s.t.

$$
\mathbf{A}_{1} \cdot \mathbf{x}_{1} \leq \mathbf{b}_{1}
$$

in which $U$ (profit,risk) is the GENCO's objective function and $\mathbf{A}_{\mathbf{1}} \cdot \mathbf{x}_{\mathbf{1}} \leq \mathbf{b}_{\mathbf{1}}$ is the constraint on GEP, i.e., (12)-(13). As shown in Fig. 1, the GEP output is sent to the lower-level problem, which is the market clearing simulation (29).

$$
\min c^{T} x
$$

s.t.

$$
\mathbf{A}_{2} \cdot \mathbf{x}_{2} \leq \mathbf{b}_{2}
$$

Here, $\mathrm{c}^{T} \cdot \mathrm{x}$ is the objective function for the market clearing, i.e., (14) and $\mathbf{A}_{\mathbf{2}} \cdot \mathbf{x}_{\mathbf{2}} \leq \mathbf{b}_{\mathbf{2}}$ is the generalized set of constraints for the market clearing model, i.e., (15)-(27).

\section{CASE STUDIES}

A six-bus system is considered to demonstrate the effectiveness of the proposed approach for solving the strategic GEP. Fig. 3 depicts the single-line diagram where $G_{g n}$ represents the existing units of GENCO $g$ located at bus $n$. Each GENCO has two existing and four candidate units. Candidate generating units have a variety of options in the site location, unit type, capacity, cost, and forced outage rate. We consider three wind zones. Each zone represents one class of wind (i.e., zones differ in wind speeds and angles for wind generators). It is assumed that Zone 1 has the best wind quality, while a wind unit in Zone 3 has the lowest wind generation capacity factor. The volatility of wind generation in each zone is considered via multiple scenarios. Hourly available wind generation is based on the historical data for each zone.

The Monte Carlo simulation method is used to create 1000 scenarios. Generating units and transmission line availability, load forecast errors, and wind speed volatilities are modeled in multiple scenarios. The scenario reduction method is used to reduce the number of scenarios from 1000 to 10 as a trade-off between the computation time and the solution accuracy [26]-[29].

The GENCOs information is listed in Tables 1 and 2. As shown in Fig. 3, loads are located at buses 3, 4, and 5. It is assumed that load 1 is the most critical one and load 3 has the least serving priority. As a result of such assumption, load 1 has the highest load shedding cost (i.e., $\$ 500 / \mathrm{MWh}$ ) and load 3 has the lowest one (i.e., $\$ 300 / \mathrm{MWh}$ ). In other words, load 3 is economically the first candidate for load shedding. The one-year average load and load shedding costs are listed in Table 3. Transmission network data is listed in Table 4. Hourly load and wind data and scenario information are provided in http://motor.ece.iit.edu/data/Hourly_Data.xls. The financial incentive for wind generation is $\alpha_{i g t}=5 \$ / \mathrm{MWh}$ and the average 
emission charge is $E C_{i g t}=5 \$ / \mathrm{MWh}$. Two case studies are considered here.

\section{Case 1: Generation Expansion Planning}

In this case, GEP is considered without any transmission network upgrades in the area. Each GENCO has the complete information of its own expansion alternatives and maximizes its utility value by making assumptions on opponents' strategies. Each strategy is associated with one of GENCO $i$ 's strategy options listed in Table 2. Table 5 shows the results for two GENCOs with four expansion strategies $\left(\mathrm{St}_{1}-\mathrm{St}_{4}\right)$. The selected strategy is associated with the highest utility value shown in bold. The results indicate that GENCO 1 selects $\mathrm{St}_{1}$ to install a thermal unit at bus 1 . GENCO 2 would prefer to install a wind generator at bus $1\left(\mathrm{St}_{1}\right)$. GENCO 2 does not pick the option to install a wind unit at bus $6\left(\mathrm{St}_{4}\right)$ despite its lower investment cost than that at bus 1 . The reason is that the wind in Zone 1 shows the best pattern and provides a higher expected payoff. This reveals one of the major differences when considering sustainable generation in the game model. When GENCOs consider conventional generators, the major merit would be the least investment and operation costs. However, as shown in this case, volatility of wind generation poses a more complicated GEP model which is solved in this paper by integrating stochastic programming in the game model.

Another interesting result is that GENCO 2 obtains a much higher payoff by preferring the wind generation option $\left(\mathrm{St}_{1}\right)$ to the cheaper investment of thermal units $\left(\mathrm{St}_{2}\right)$. This case is of not only the low operating cost, available incentives payment, low emission, and risk-free fuel for sustainable generating units, but also the favorable wind speed in Zone 1. Sustainable generation resources usually incur high initial investments and volatile power output. However, participants can still benefit from such resources by making proper pre-expansion studies on sites, technologies, and market situation.

\section{Case 2: Transmission-Constrained Generation Expansion}

In this case, GENCOs make GEP investment decisions while considering possible transmission network expansions in the market simulation. Similar to Case 1, each GENCO has four strategies while considering four expected transmission expansion strategies listed in Table 6. Therefore, each GENCO would evaluate 64 options in order to select the final plan. For a large number of GENCOs and wide ranges of strategies, the GEP problem might become very complicated and time consuming. The parallel computing applied to scenarios could reduce the CPU time that is about 300 seconds. Table 7 includes results of transmission-constrained GEP. The results of transmission-constrained GEP indicate that GENCO 2 is not expected to change its strategy in Case 2. However, its payoff is reduced when transmission constraints are introduced. This concept is a major issue in the transmission-constrained GEP. In practice, under certain conditions, a candidate generating unit may be substituted by an additional transmission capacity for enhancing the payoff.

The social welfare in Tables 5 and 7 is calculated as the difference between load payments and dispatch costs. The highest 
social welfare in different cases is associated with sustainable generation. This is because these units impose smaller operating costs (i.e. production, outage, and emission costs) or equivalently a higher social welfare for the benefit of the entire system. In Case 2, a considerable saving in social welfare is due to transmission-constrained GEP. This example shows the effectiveness of the proposed model for a holistic simulation of GEP while exploring the value of GEP to the transmission-constrained system security.

Table 8 shows the impact of emission cost variation on GENCOs' strategies. Here, GENCO 2 has planned a wind unit even when the emission cost is zero. When the emission penalty cost is higher, the thermal unit options will start losing revenues. Table 9 shows GENCOs' strategies as a function of fuel price. The standard deviation of fuel price is set at a moderate level of $10 \%$ of forecasted price; however, a $30 \%$ or higher volatility in fuel price is plausible. We assume that the existing generating units would have long-term fuel contracts, which are not affected by fuel price volatilities. The wind unit has a low operating cost which is not subject to fuel price uncertainty. Table 8 shows that fuel price volatilities do not affect the GENCO g's payoff when considering wind units. However, higher fuel price volatilities could heighten the tendency to install additional wind units. This observation would justify the importance of considering financial risks in investment analyses.

Table 10 shows that GENCO's utility value may be sensitive to capacity limits of transmission lines. For instance, in the case of GENCO 1, the utility value drops by about $48 \%$ when the transmission capacity increases from $100 \mathrm{MW}$ to $250 \mathrm{MW}$. This is because the GENCO 1's high LMPs at bus 1 ( $\mathrm{St}_{1}$ of GENCO 1) would drop if the new transmission capacity is added to the system. This approach would help GENCOs avoid misleading high expected outcomes.

\section{CONCLUSiOnS}

Strategic generation expansion planning model from market participants' viewpoint is proposed in this paper by considering volatile sustainable energy resources. Utility value concept is utilized which would effectively model entities' profit over the planning horizon while taking into account the cost of uncertain factors. The proposed approach provides the planner with additional information on the investment return for certain market price forecasts, which will be helpful for the investment decision-making.

Two observations are made based on these results. First, the GENCO ought to make stochastic expansion analyses with the volatile sustainable energy sources while considering other participants' roles for securing its financial return. Second, using transmission-constrained generation expansion planning contributes to avoid unrealistic payoff for the GENCO.

The case studies in general present the effectiveness of the proposed planning approach. The proposed method considers the transmission-constrained GEP model, in which the GENCO simulates the simultaneous energy and ancillary market operations to maximizing its payoff while considering other GENCOs generation expansion strategies.

The salient feature of the proposed method is that it includes sustainable energy resources as a suitable alternative for GEP to 
reduce the risk of price uncertainty in fuel and emission. The results show that despite higher investment costs, sustainable options can compete with conventional energy sources if proper placement and penetration level studies are done before installation. Since the number of scenarios considered can grow exponentially based on numbers of the GENCO's strategies and its opponents, the simulation time may escalate significantly. This issue can be mitigated using top-ranked situations yield from branch-and-bound-based method instead of considering all possible combinations in each game iteration. This subject will be more explored in our future work.

\section{ACKNOWLEDGMENT}

This study is funded in part by the U.S. Department of Energy Grants \# DE-EE 0002979 and DE-EE 0001380.000.

\section{REFERENCES}

[1] M. Shahidehpour, Z. Li, Y. Fu, Energy System, John Wiley and Sons, 2012.

[2] Y. Fu, M. Shahidehpour, Profit-based generation resource planning, IMA J. of Management Mathematics (Oxford University Press), 15 (4) (2004) 273-289.

[3] J.H. Roh, M. Shahidehpour, Y. Fu, Security-constrained resource planning in electricity markets, IEEE Trans. Power Syst. 22 (2) (2007) 812-820.

[4] E. Gnansounou, J. Dong, S. Pierre A. Quintero, Market Oriented Planning of Power Generation Expansion using Agentbased Model, IEEE/PES Power System Conference and Exposition (PSCE), 3 (2004) 1306-1311.

[5] A.S. Chuang, F. Wu, P. Varaiya, A game-theoretic model for generation expansion planning: problem formulation and numerical comparisons, IEEE Trans. Power Syst., 16 (4) (2001) 885-891.

[6] J. Wang, M. Shahidehpour, Z. Li, A. Botterud, Strategic generation capacity expansion planning with incomplete information, IEEE Trans. Power Syst., 24 (2) (2009) 1002-1010.

[7] S. K. K. Ng, J. Zhong, C. W. Lee, A game-theoretic study of the strategic interaction between generation and transmission expansion planning, IEEE/PES Power System Conference and Exposition (PSCE), (2009) 1-10.

[8] A. Botterud, M. R. Mahalik, T.D. Veselka, R. Heon-Su, S. Ki-Won, Multi-agent simulation of generation expansion in electricity Markets, IEEE PES General Meeting 2007; 1-8.

[9] H. A. Shayanfar, A. Saliminia Lahiji, J. Aghaei, A. Rabiee, Generation expansion planning in pool market: A hybrid modified game theory and improved genetic algorithm, J of Energy Conversion and Management 50 (5) (2009) 1149-1156.

[10] W. Vick, Counter speculation, auctions, and competitive sealed tenders, J of Finance 16 (1) (1961) 8-37.

[11] T. Li, M. Shahidehpour, Strategic bidding of transmission-constrained GENCOs with incomplete information, IEEE 
Trans. Power Syst. 20 (1) (2005) 437-447.

[12] R. Ferrero, J. Rivera, M. Shahidehpour, Applications of games with incomplete information for pricing electricity in deregulated power pools, IEEE Trans. Power Syst., 13 (1) (1998) 184-189.

[13] M. R. Hesamzadeh, M. Amelin, A Generation Expansion Planning Model of a Strategic Electricity Generating Firm. IEEE PES General Meeting, (2011) 1-7.

[14] M. J. Newton, P. D. Hopewell, Costs of sustainable electricity generation, J of Engineering Science and Education, 11 (2) (2002) 49-55.

[15] D. A. Balser, Sustainable generation portfolio, IEEE Power Engineering Review, 22 (5) (2002) 12-15.

[16] C. Unsihuay-Vila, J. W. Marangon-Lima, A. C. Zambroni de Souza, I. J. Perez-Arriaga, Multistage expansion planning of generation and interconnections with sustainable energy development criteria: A multiobjective model, Int. J of Electrical Power and Energy Systems, 33 (2) (2011) 258-270.

[17] B. Cai, Y. Xue, F. Wen, J. Liu, W. Li, Hybrid Simulation of Generation and Transmission Planning in Market Environment. IEEE 4th Int. conf. on Electric Utility Deregulation and Restructuring and Power Technologies (DRPT), (2011) 128-133.

[18] S. Awerbuch, Valuing renewable and conventional generating assets in an environment of uncertainty and technological change, Environmental Audit Committee, House of Commons, (2005).

[19] F. Delgado, A. Ortiz, C. J. Renedo, S. Pérez, M. Mañana, A. F. Zobaa, The influence of nuclear generation on CO2 emissions and on the cost of the Spanish system in long-term generation planning, Int. J of Electrical Power and Energy Systems, 33 (3) (2011) 673-683.

[20] J. Lei, H. Xin, J. Xie, D. Gan, Optimization of distributed energy systems taking into account energy saving and emission reduction. Int. Conf. on Sustainable Power Generation and Supply (SUPERGEN), (2009) 1-6.

[21] J. E. Platts, Impact of regional green-house gas initiative and renewable portfolio standards on power system planning, IEEE Power Engineering Society General Meeting, (2006).

[22] A. G. Bakirtzis, A probabilistic method for the evaluation of reliability of stand-alone wind energy system, IEEE Trans. Energy Conv., 7 (1) (1997) 99-107.

[23] S. A. Farghal, M. R. Abdel Aziz, Generation expansion planning including the renewable energy sources. IEEE Trans. Power Syst., 3 (3) (1998) 816-822.

[24] J. Valenzuela, J. Wang, A probabilistic model for assessing the long-term economics of wind energy, Elsevier Electric Power System Research, 81 (4) (2011) 853-861.

[25] X. Bai, M. Shahidehpour, V. C. Ramesh, Y. Erkeng, Transmission Analysis by Nash Game Method, IEEE Trans. Power 
Syst. 12 (3) (1997) 1046-1052.

[26] L. Wu L, M. Shahidehpour, T. Li, Stochastic security-constrained Unit commitment, IEEE Trans. Power Systems, 22 (2) (2007) 800-811.

[27] S. Kamalinia, M. Shahidehpour, Generation expansion planning of wind-thermal in power systems, IET Gener. Transm. Distrib. 4 (8) (2010) 940-951.

[28] A. Khodaei, M. Shahidehpour, S. Kamalinia, Transmission switching in expansion planning, IEEE Trans. Power Syst., 25 (3) (2010) 1722-1733.

[29] S. Kamalinia, M. Shahidehpour, A. Khodaei, Security-Constrained Expansion Planning of Fast-Response Units for Wind Integration, Elsevier Electric Power System Research, 81 (1) (2011) 107-116.

[30] C. Novoa, T. Jin, Reliability centered planning for distributed generation considering wind power volatility, Int. J of Electrical Power and Energy Systems, 81 (8) (2011) 1654-1661.

[31] Z. Bodie, A. Kane, J. Marcus, Investments, seventh ed. McGraw-Hill Irwin International Edition, 2008.

[32] J. P. S. Catalão, S. J. P. S. Mariano, V. M. F. Mendes, L. A. F. M. Ferreira, A practical approach for profit-based unit commitment with emission limitations, Int. J of Electrical Power and Energy Systems, 32 (3) (2010) 218-224.

[33] S. Kamalinia, M. Shahidehpour, Capacity adequacy calculation by considering locational capacity prices, IET Gener. Transm. Distrib., 4 (3) (2010) 376-385.

[34] J. Wang, M. Shahidehpour, Z. Li, Security-constrained unit commitment with volatile wind power generation, IEEE Trans. Power Syst., 23 (3) (2008) 1319-1327. 
Figure Captions:

Fig. 1 Framework for the GENCOs' GEP

Fig. 2 Utility Function for Different Degrees of Participants' Risk Aversion

Fig. 3 One-Line Diagram of Six-Bus System

Figure 


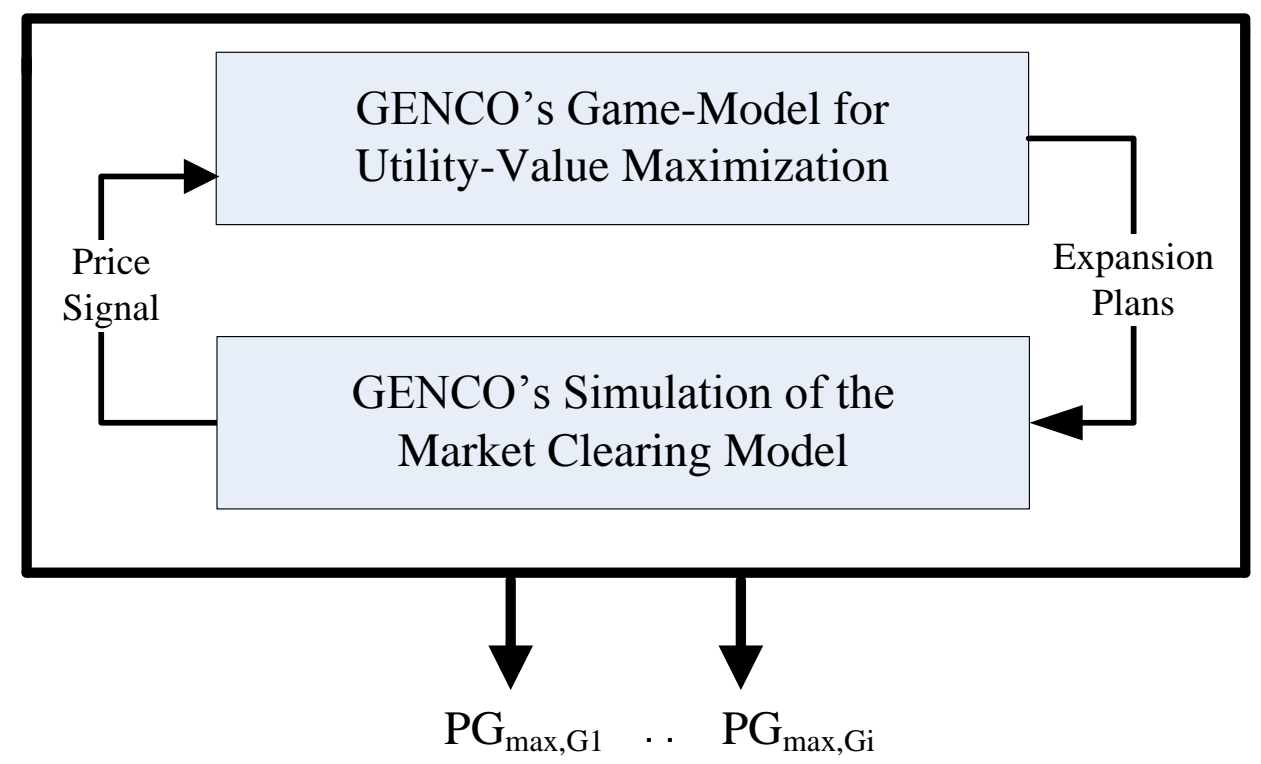

Fig. 1. Framework for the GENCOs' GEP

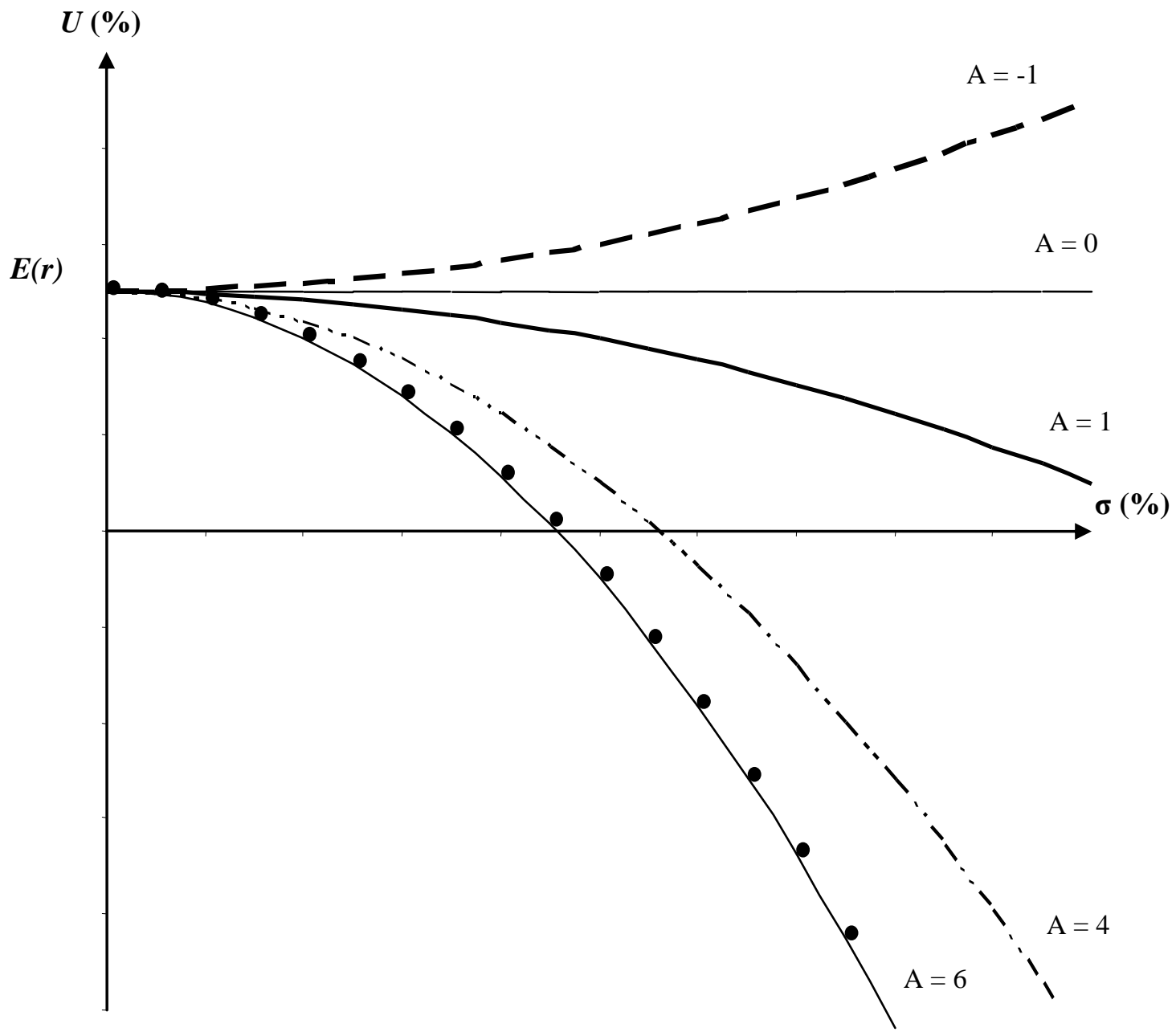

Fig. 2. Utility function for different degrees of participants' risk aversion 


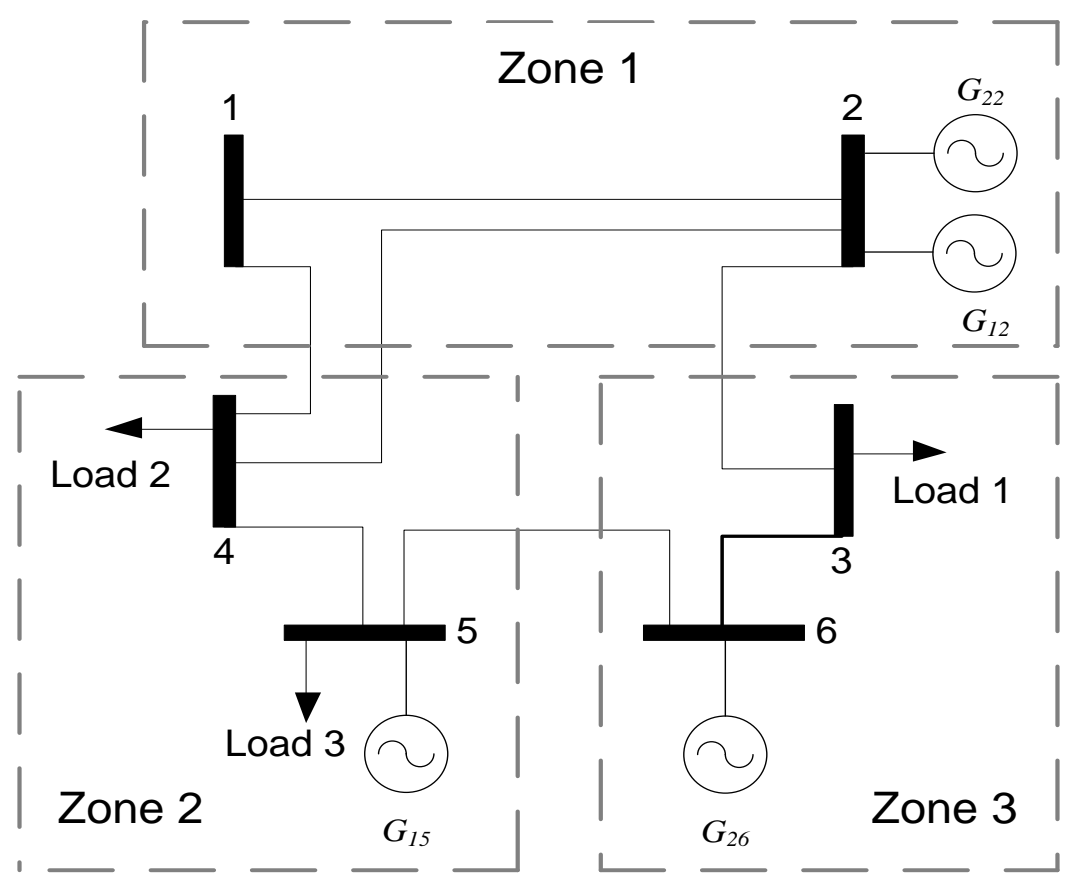

Fig. 3. One-Line Diagram of the Six-Bus System 
Table Captions:

Table 1 Existing Unit Data

Table 2 Candidate Unit Data

Table 3 Load Quantities and Load Shedding Costs

Table 4 Six- Bus System Transmission System Data

Table 5 Results of Various GEP Strategies

Table 6 Transmission Expansion Data

Table 7 Transmission-Constrained GEP

Table 8 GENCO' Payoff Sensitivity to Emission Costs

Table 9 GENCO’ Payoff Sensitivity to Fuel Price

Table 10GENCO' Payoff Sensitivity to Fuel Price with Transmission Constraints 
Table 1 Existing Unit Data

\begin{tabular}{ccccccc}
\hline \hline Bus & Unit & Operation Cost & Emission Cost & Capacity & FOR & GENCO \\
Index & Type & $(\$ / \mathrm{MWh})$ & $(\$ / \mathrm{MWh})$ & $(\mathrm{MW})$ & $(\%)$ & Index \\
\hline 2 & Thermal & 52.0 & 5 & 450 & 7 & 1 \\
5 & Thermal & 45.6 & 5 & 350 & 7 & 1 \\
2 & Thermal & 59.3 & 5 & 450 & 7 & 2 \\
6 & Thermal & 35.5 & 5 & 350 & 8 & \\
\hline \hline
\end{tabular}

Table 2 Candidate Unit Data

\begin{tabular}{ccccccccc}
\hline \hline Bus & Unit Type & $\begin{array}{c}\text { Investment Cost } \\
\text { Index }\end{array}$ & $\begin{array}{c}\text { Operation Cost } \\
(\$ \mathrm{k} / \mathrm{MW} / \mathrm{year})\end{array}$ & $\begin{array}{c}\text { Emission Cost } \\
(\$ / \mathrm{MWh})\end{array}$ & $\begin{array}{c}\text { Capacity } \\
(\$ / \mathrm{MWh})\end{array}$ & $\begin{array}{c}\text { FOR } \\
(\mathrm{MW})\end{array}$ & $\begin{array}{c}\text { GENCO } \\
(\%)\end{array}$ & $\begin{array}{c}\text { Index } \\
\mathrm{St}_{\#}\end{array}$ \\
\hline 1 & Thermal & 30 & 17.1 & 5 & 300 & 7 & 1 & $\mathrm{St}_{1}$ \\
1 & Wind & 175 & 0 & 0 & 300 & 0.5 & 1 & $\mathrm{St}_{2}$ \\
4 & Wind & 165 & 0 & 0 & 350 & 0.5 & 1 & $\mathrm{St}_{3}$ \\
6 & Thermal & 24 & 29.5 & 5 & 400 & 8 & 1 & $\mathrm{St}_{4}$ \\
1 & Wind & 175 & 0 & 0 & 300 & 0.5 & 2 & $\mathrm{St}_{1}$ \\
1 & Thermal & 30 & 15.7 & 5 & 350 & 4 & 2 & $\mathrm{St}_{2}$ \\
5 & Thermal & 31 & 31.4 & 5 & 350 & 7 & 2 & $\mathrm{St}_{3}$ \\
6 & Wind & 163 & 0 & 0 & 350 & 0.5 & 2 & $\mathrm{St}_{4}$ \\
\hline \hline
\end{tabular}

Table 3 Load Quantities and Load Shedding Costs

\begin{tabular}{cccc}
\hline \hline Load Number & Bus & Annual Average Quantity (MW) & Load Shedding Cost (\$/MWh) \\
\hline 1 & 3 & 500 & 500 \\
2 & 4 & 250 & 400 \\
3 & 5 & 750 & 300 \\
\hline \hline
\end{tabular}


Table 4 Six- Bus System Transmission System Data

\begin{tabular}{ccccc}
\hline \hline Line Number & From Bus & To Bus & X (pu) & Capacity Limit (MW) \\
\hline 1 & 1 & 2 & 0.170 & 250 \\
2 & 1 & 4 & 0.258 & 250 \\
3 & 2 & 4 & 0.197 & 250 \\
4 & 5 & 6 & 0.140 & 250 \\
5 & 2 & 3 & 0.237 & 250 \\
6 & 4 & 5 & 0.237 & 250 \\
7 & 3 & 6 & 0.218 & 250 \\
\hline \hline
\end{tabular}

Table 5 Results of Various GEP Strategies

\begin{tabular}{|c|c|c|c|c|c|c|c|c|}
\hline \multirow{2}{*}{ Results } & \multicolumn{4}{|c|}{ GENCO 1} & \multicolumn{4}{|c|}{ GENCO 2} \\
\hline & St1 & St2 & St3 & St4 & St1 & St2 & St3 & St4 \\
\hline Utility Value (\$M) & 32.80 & 32.38 & 26.06 & -8.40 & 44.74 & 30.51 & -0.40 & 23.8 \\
\hline $\begin{array}{l}\text { Average Expected Social Welfare } \\
\qquad(\$ B)\end{array}$ & 2.65 & 2.81 & 2.02 & 0.96 & 3.66 & 3.23 & 1.18 & 2.60 \\
\hline
\end{tabular}

Table 6 Transmission Expansion Data

\begin{tabular}{ccc}
\hline \hline From Bus Number & To Bus Number & Capacity (MW) \\
\hline 1 & 5 & 250 \\
2 & 6 & 250 \\
3 & 5 & 250 \\
3 & 4 & 250 \\
\hline \hline
\end{tabular}


Table 7 Transmission-Constrained GEP

\begin{tabular}{cccc}
\hline \hline Participant & Strategy & Utility Value $(\$ \mathrm{M})$ & Expected Social Welfare $(\$ \mathrm{~B})$ \\
\hline \multirow{2}{*}{ GENCO 1 } & $\mathrm{St}_{1}$ & 17.83 & 1.54 \\
& $\mathrm{St}_{2}$ & 16.53 & 1.69 \\
& $\mathrm{St}_{3}$ & 20.21 & 1.24 \\
& $\mathrm{St}_{4}$ & -7.48 & 0.93 \\
\hline & $\mathrm{St}_{1}$ & 24.15 & 2.29 \\
& $\mathrm{St}_{2}$ & 11.94 & 1.85 \\
& $\mathrm{St}_{3}$ & -0.97 & 0.87 \\
& $\mathrm{St}_{4}$ & 13.80 & 2.19 \\
\hline \hline
\end{tabular}

Table 8 GENCO' Payoff Sensitivity to Emission Costs

\begin{tabular}{|c|c|c|c|c|c|c|c|}
\hline & & $\mathrm{EC}=0$ & $\mathrm{EC}=5$ & $\overline{\mathrm{EC}=10}$ & $\overline{\mathrm{EC}}=15$ & $\mathrm{EC}=20$ & $\mathrm{EC}=25$ \\
\hline GENCO 1's Max Utility Value & $\mathrm{G}^{(\mathrm{a})}$ & $\begin{array}{l}43.67 \\
{\left[\mathrm{St}_{1}\right]}\end{array}$ & $\begin{array}{r}32.8 \\
{\left[\mathrm{St}_{1}\right]}\end{array}$ & $\begin{array}{r}24.67 \\
{\left[\mathrm{St}_{2}\right]}\end{array}$ & $\begin{array}{l}16.96 \\
{\left[\mathrm{St}_{2}\right]}\end{array}$ & $\begin{array}{l}9.25 \\
\\
{\left[\mathrm{St}_{2}\right]}\end{array}$ & $\begin{array}{l}1.54 \\
{\left[\mathrm{St}_{2}\right]}\end{array}$ \\
\hline$\left[\right.$ Best $\left.\mathrm{St}_{\#}\right](\$ \mathrm{M})$ & $\mathrm{G} \& \mathrm{~T}^{(\mathrm{b})}$ & 28.76 & 20.21 & 13.4 & $\begin{array}{r}6.6 \\
{\left[\mathrm{St}_{2}\right]}\end{array}$ & -0.2 & -7.02 \\
\hline GENCO 2's Max Utility Value & G & $\begin{array}{l}49.9 \\
{\left[\mathrm{St}_{1}\right]}\end{array}$ & $\begin{array}{l}44.74 \\
{\left[\mathrm{St}_{1}\right]}\end{array}$ & $\begin{array}{l}39.59 \\
{\left[\mathrm{St}_{1}\right]}\end{array}$ & $\begin{array}{l}34.42 \\
{\left[\mathrm{St}_{1}\right]}\end{array}$ & $\begin{array}{r}29.27 \\
{\left[\mathrm{St}_{1}\right]}\end{array}$ & $\begin{array}{r}24.11 \\
{\left[\mathrm{St}_{1}\right]}\end{array}$ \\
\hline$\left[\right.$ Best $\left.\mathrm{St}_{\#}\right](\$ \mathrm{M})$ & $\mathrm{G} \& \mathrm{~T}$ & $\begin{array}{c}29.42 \\
{\left[\mathrm{St}_{1}\right]}\end{array}$ & $\begin{array}{r}24.15 \\
{\left[\mathrm{St}_{1}\right]}\end{array}$ & $\begin{array}{r}18.88 \\
{\left[\mathrm{St}_{1}\right]}\end{array}$ & $\begin{array}{l}13.6 \\
{\left[\mathrm{St}_{1}\right]}\end{array}$ & $\begin{array}{l}8.33 \\
{\left[\mathrm{St}_{1}\right]}\end{array}$ & $\begin{array}{l}3.06 \\
{\left[\mathrm{St}_{1}\right]}\end{array}$ \\
\hline
\end{tabular}

(a) G: Generation planning (no transmission constraints)

(b) G \& T: Transmission-constrained generation planning 
Table 9 GENCO' Payoff Sensitivity to Fuel Price

\begin{tabular}{|c|c|c|c|c|c|c|c|}
\hline & & $\sigma=0$ & $\sigma=5$ & $\sigma=15$ & $\sigma=20$ & $\sigma=25$ & $\sigma=30$ \\
\hline & & 35.05 & 34.48 & 32.37 & 32.37 & 32.37 & 32.37 \\
\hline GENCO 1's Max Utility Value & & {$\left[\mathrm{St}_{1}\right]$} & {$\left[\mathrm{St}_{1}\right]$} & {$\left[\mathrm{St}_{2}\right]$} & {$\left[\mathrm{St}_{2}\right]$} & {$\left[\mathrm{St}_{2}\right]$} & {$\left[\mathrm{St}_{2}\right]$} \\
\hline \multirow[t]{2}{*}[\text{Best}\mathrm{St}_{\#}]{$(\$ \mathrm{M})$} & \multirow{2}{*}{$\mathrm{G} \& \mathrm{~T}$} & 20.21 & 20.21 & 20.21 & 20.21 & 20.21 & 20.21 \\
\hline & & {$\left[\mathrm{St}_{3}\right]$} & {$\left[\mathrm{St}_{3}\right]$} & {$\left[\mathrm{St}_{3}\right]$} & {$\left[\mathrm{St}_{3}\right]$} & {$\left[\mathrm{St}_{3}\right]$} & {$\left[\mathrm{St}_{3}\right]$} \\
\hline \multirow{4}{*}{ GENCO 2's Max Utility Value } & \multirow{2}{*}{ G } & 44.74 & 44.74 & 44.74 & 44.74 & 44.74 & 44.74 \\
\hline & & {$\left[\mathrm{St}_{1}\right]$} & {$\left[\mathrm{St}_{1}\right]$} & {$\left[\mathrm{St}_{1}\right]$} & {$\left[\mathrm{St}_{1}\right]$} & {$\left[\mathrm{St}_{1}\right]$} & {$\left[\mathrm{St}_{1}\right]$} \\
\hline & \multirow{2}{*}{$\mathrm{G} \& \mathrm{~T}$} & 25.06 & 24.15 & 24.15 & 24.15 & 24.15 & 24.15 \\
\hline & & {$\left[\mathrm{St}_{2}\right]$} & {$\left[\mathrm{St}_{1}\right]$} & {$\left[\mathrm{St}_{1}\right]$} & {$\left[\mathrm{St}_{1}\right]$} & {$\left[\mathrm{St}_{1}\right]$} & {$\left[\mathrm{St}_{1}\right]$} \\
\hline
\end{tabular}

Table 10 GENCO' Payoff Sensitivity to Fuel Price with Transmission Constraints

\begin{tabular}{cccccc}
\hline \hline & PLC=100 (MW) & PLC=250 (MW) & PLC=350 (MW) & PLC=500 (MW) & PLC=650 (MW) \\
\hline GENCO 1's Utility Value & 38.95 & 20.21 & 20.4 & 20.4 & 20.4 \\
{$[$ Best St $\#$ (\$M) } & {$\left[\mathrm{St}_{1}\right]$} & {$\left[\mathrm{St}_{3}\right]$} & {$\left[\mathrm{St}_{1}\right]$} & {$\left[\mathrm{St}_{1}\right]$} & {$\left[\mathrm{St}_{1}\right]$} \\
\hline GENCO 2's Utility Value & 26.6 & 24.15 & 27.16 & 27.19 & 27.19 \\
{$[$ Best St } & & & & \\
\hline
\end{tabular}

